\title{
Inhibidores para emprender que pueden atenderse desde un enfoque de orientación para la carrera: el caso de los estudiantes emprendedores de la UNED
}

Luis Miguel Almagro-Gavira

UNED, C. A. de Sevilla (España)

Nuria Manzano-Soto

UNED (España) 



\title{
Inhibidores para emprender que pueden atenderse desde un enfoque de orientación para la carrera: el caso de los estudiantes emprendedores de la UNED
}

\section{Inhibitors to undertake that can be addressed from a career orientation approach: the case of the entrepreneurial students of the UNED}

\author{
Luis Miguel Almagro-Gavira \\ UNED, C. A. de Sevilla (España) \\ lmalmagro@sevilla.uned.es \\ Nuria Manzano-Soto \\ UNED (España) \\ nmanzano@edu.uned.es
}

Fecha de recepción: 28 de septiembre de 2020

Fecha de aceptación: 22 de diciembre de 2020

\section{Resumen}

En los últimos años se han multiplicado los programas orientados a la formación de emprendedores y el fomento de la cultura e iniciativa emprendedora, aunque la evaluación de estos servicios y programas no ha recibido la misma atención. En este trabajo se presentan los primeros resultados de un estudio exploratorio sobre las barreras que dificultan el emprendimiento y cómo abordarlos desde la orientación para la carrera. En concreto se estudia el programa de emprendimiento universitario llevado a cabo en el Centro de Orientación y Empleo (COIE) de la Universidad Nacional de Educación a Distancia (UNED). Los resultados obtenidos identifican los inhibidores para emprender y los motivos que tienen los emprendedores universitarios noveles para el abandono de sus proyectos de emprendimiento. Asimismo, se presentan los aspectos personales de mejora identificados por los promotores de proyectos para continuar con sus iniciativas, y algunas propuestas 
para la mejora de los servicios de orientación a emprendedores que sus usuarios manifiestan.

Palabras clave: Emprendimiento; Orientación profesional; Inhibidores para emprender; Programas de orientación y emprendimiento.

\begin{abstract}
In recent years, programmes aimed at training entrepreneurs and promoting culture and entrepreneurial initiative have multiplied, although the evaluation of these services and programmes has not received the same attention. This paper presents the first results of an exploratory study on the barriers that hinder entrepreneurship and how to approach them from career guidance. Specifically, the university entrepreneurship programme carried out at the Counselling and Employment Center (COIE) of The National Distance Education University (UNED) is studied. The results obtained identify the inhibitors to entrepreneurship and the reasons that beginner's university entrepreneurs have for revoking their entrepreneurship projects. Likewise, the personal aspects of improvement identified by the project promoters are presented to continue with their initiatives, and some proposals for the improvement of the guidance services for entrepreneurs that their users manifest.
\end{abstract}

Keywords: Entrepreneurship; Career guidance; Entrepreneurship inhibitors; Guidance and entrepreneurship programmes.

\title{
1. INTRODUGGIÓN
}

En la actualidad parece haber consenso en la consideración del término emprender como el desarrollo de un conjunto de acciones de creación e innovación para generar valor en un ámbito determinado (Alda, 2010). El proceso emprendedor incluye actividades que van desde la exploración del entorno y la generación de una idea, hasta la materialización de una iniciativa emprendedora (Alda-Varas et al., 2012). Desde esta perspectiva, el emprendimiento no solo se concibe como la creación de una empresa, sino como un modo de pensar y actuar, es decir, un individuo puede convertir las ideas en acciones y ser más creativo y seguro de sí mismo en lo que emprenda (Kirby e Ibrahim, 2011).

Asimismo, se comprende que "el emprendimiento no es sólo una alternativa al desempleo", es "empleo que genera empleo y que genera economía, la transforma y aumenta su competitividad" (Amor, 2016, p.76). Así, durante la última década la situación de incertidumbre económica y laboral está originando que las personas afectadas por el desempleo cambien la forma de afrontar y entender su inserción laboral y opten por vías alternativas al trabajo por cuenta ajena, incrementándose la opción del emprendimiento (entendido como autoempleo y/o creación de empresas), para el que se requiere un empoderamiento socio personal (Suarez, Cortés y Sánchez, 2015).

La concepción del autoempleo y la creación de empresas como importante motor de crecimiento económico, junto a las recomendaciones europeas sobre la pro- 
moción del emprendimiento (Comisión EU, 2012; Consejo EU, 2013) han provocado tanto una mayor implicación por el fomento de la iniciativa emprendedora, como una mayor relevancia del concepto de emprendimiento, llegando a considerarse una solución para el freno del problema del desempleo (Estrategia Española de Activación para el Empleo, 2017-2020), de forma que ha tomado más fuerza que nunca la idea de incentivar el emprendizaje en todas las esferas y niveles sociales.

La literatura científica también promueve el emprendimiento, son cada vez más comunes las contribuciones metodológicas y estudios sobre prácticas pedagógicas asociadas al mismo (e.g. Neck y Greene, 2011). Hay consenso en afirmar que las competencias emprendedoras se pueden aprender (Chell, 2013; García et al., 2013 y Torrejón, 2016) y "si bien pueden existir unas pocas personas que de forma innata dispongan de una combinación de competencias idóneas para el desarrollo empresarial y la iniciativa de negocio, con carácter general las competencias y sus atributos son educables y perfeccionables" (Torrejón, 2016, p. 45).

El estudio de las competencias emprendedoras es complejo (ver tabla 1) y se puede abordar desde dos perspectivas: una basada en el desarrollo de ciertas cualidades personales y otra centrada directamente en la generación de empresas (Sobrado y Fernández, 2010).

Hornaday (1971, cit. por Torrejón, 2016) propuso una serie de competencias emprendedoras, aceptadas actualmente y ampliadas por distintos autores. Estas son: autoconfianza y optimismo, habilidad para asumir riesgos calculados, respuesta positiva a los retos, flexibilidad y adaptabilidad, conocimiento de los mercados, buenas relaciones interpersonales, energía y disposición, creatividad y motivación de logro, liderazgo y receptividad a las opiniones. Boyles (2012) señala como elementos críticos la capacidad creativa e innovadora, la disposición para asumir riesgos, la capacidad de resolver problemas, la iniciativa, el liderazgo, la habilidad de trabajo en equipo y de comunicación. Por su parte, Mets et al. (2017) agregan las habilidades de gestión de proyectos, el manejo de la incertidumbre y actitudes como autonomía, tolerancia al fracaso, autoestima y autoeficacia. Por otro lado, en lo que se refiere a los conocimientos, los mismos autores destacan la necesidad de aprender a elaborar planes de empresas, desarrollar modelos de negocios, procesos de emprendimiento y aspectos de finanzas y marketing, entre otros.

Los diversos atributos de la competencia emprendedora hacen visible la complejidad que supone el perfil personal para enfrentarse al autoempleo con ciertas garantías. Sin embargo, las teorías que afirman que las competencias son educables, motivan la creencia en la posibilidad real de emprender tras aprender a hacerlo.

Así, la promoción del emprendimiento consigue que individuos que a priori son más reacios a lanzarse a la aventura de emprender se animen a hacerlo, de forma que a muchas personas con dificultad para emplearse por cuenta ajena y que en otras condiciones nunca se lo hubiesen planteado, considerados emprendedores por 
necesidad (Block y Wagner, 2010; Peña, et al., 2016), se despierta su interés por emprender. En el grupo de pre-emprendedores por necesidad, habitualmente con altos grados de desinformación al respecto y sin plena confianza en sus posibilidades para llegar a convertir sus ideas en realidades empresariales, en caso de tenerlas, existe un elevado número de futuros emprendedores vulnerables (Manzano y Almagro, 2016).

En cuanto a los distintos perfiles de emprendedores -emprendedor por oportunidad vs emprendedor por necesidad-sabemos que los segundos en mayor medida que los primeros se enfrentan al emprendimiento con temor debido principalmente a dos razones: 1) creer en la posibilidad de empeorar su situación económica en el caso de no conseguir el éxito de sus iniciativas, y 2) la "incapacidad" auto-percibida por no considerarse a sí mismos emprendedores 'de nacimiento' (Almagro y Manzano, 2017). Este temor condiciona la puesta en marcha y el funcionamiento de los proyectos, pero también la elección de los mismos pues este tipo de emprendedores optan por proyectos de bajo coste y una rápida implementación. Esto tiene una contrapars tida negativa pues la elección y desarrollo de ese tipo de oportunidades de negocio permite explicar las diferencias de crecimiento y desarrollo de las nuevas empresas (Liñan, et al., 2013). En este sentido, Carsrud y Brännback (2011) afirman que los emprendedores por necesidad se ven forzados a emprender para superar las barreras externas que les dificultan la incorporación al mercado laboral, y por tanto se dirigen a emprender proyectos a veces menos innovadores u orientados al crecimiento que tienen un periodo de recuperación más largo (Aido et al., 2016).

Sin embargo, los emprendedores por oportunidad, pueden experimentar la situación contraria. Es decir, suelen exhibir un comportamiento proactivo de búsqueda y reconocimiento de oportunidades reales de negocio de forma que anteponen la iniciativa emprendedora a otras opciones atractivas de trabajo por cuenta ajena (Peña et al., 2016). Además, los proyectos empresariales creados por oportunidad presentan mayores índices de supervivencia, aspiraciones de crecimiento y una mayor incidencia sobre la creación de empleo y la innovación (Liñan et al., 2013), por tanto, tienen más opciones de generar empresas de alto potencial (Sserwanga y Rooks, 2013) a diferencia de los emprendimientos generados por necesidad.

En este sentido, se observa que existen diferencias en la posibilidad del éxito emprendedor según el punto de partida.

Se han estudiado también aspectos relacionados con la auto-percepción que el emprendedor tiene sobre su capacidad emprendedora. Por ejemplo, se conoce la importancia de la motivación como motor del emprendimiento y del compromiso (Bayonet al. (2015) y Uy et al. (2015). Es decir, se afirma que la motivación del emprendedor con su proyecto condiciona el esfuerzo que él mismo está dispuesto a hacer para iniciarlo y mantenerlo. Pero es posible que el punto de partida (necesidad vs oportunidad) también afecte a la motivación, pues se ha identificado que la motivación de una persona para convertirse en emprendedor se basa en la probabilidad subjetiva de que sus esfuerzos sean seguidos por un resultado específico y de interés, 
así como que la motivación por emprender depende de la percepción de lograr resultados deseables (Barba-Sánchez y Atienza-Sahuquillo, 2017).

Sin duda la motivación emprendedora de un individuo es importante en la decisión de involucrarse en el trabajo por cuenta propia. Holland y Garrett (2015) postulan que los proyectos emprendedores no solo los inician aquellos que tienen la capacidad para hacerlo, sino también por aquellos que tienen la motivación necesaria.

Volviendo al punto de partida, consideramos que auto-percibirse preparado (capacitado) para iniciar un proyecto es un paso previo a tener la motivación suficiente para llevarlo a cabo, y nos planteamos qué deben conocer los emprendedores sobre sí mismos para estar capacitados para emprender y qué competencias pueden entrenarse para afrontar nuevos proyectos de autoempleo y/o empresariales. Estas cuestiones deberían tenerse en cuenta a la hora de diseñar programas para promover el espíritu empresarial y/o atender a los emprendedores.

Desde nuestro punto de vista, fomentar el emprendimiento per se sin tener en cuenta los anteriores aspectos, puede provocar también el fracaso de algunos proyectos de emprendimiento debido al desconocimiento previo de las barreras, adversidades, frustraciones, errores e insatisfacciones que también están presentes durante el camino. Manzano y Almagro (2016) identifican que los factores limitantes al emprendimiento externos al emprendedor en los que no pueden incidir fácilmente son: la financiación de sus proyectos, la falta de espacios para llevar a cabo sus iniciativas, la burocracia de los trámites de constitución, la alta fiscalidad a la que se ven sometidos, los problemas derivados de desacuerdos entre socios, etc.

La dificultad más frecuente, señalada por los emprendedores, es el acceso a la financiación. Los escasos créditos a los que tienen acceso los emprendedores requieren avales personales que éstos no siempre tienen a su disposición y, en caso de tenerlos, elevan considerablemente el riesgo, muy por encima de las expectativas de los emprendedores noveles.

Por otro lado, parece existir una relación directa entre factores internos a la persona y determinados factores externos que afectan al emprendimiento. Torrejón (2016) afirma que algunas características personales de los emprendedores pueden atraer otros tipos de financiación para los proyectos. Concretamente, los inversores privados u operadores de capital riesgo se fijan en la parte soft del negocio como es la calidad del equipo emprendedor para entrar en la operación, más allá de los habituales indicadores analizados por el capital riesgo para entrar en el capital de una sociedad (económicos, financieros, técnicos, legales, etc.).

En esta misma línea Knockaert y Wright (2010), analizaron cómo las empresas de capital riesgo se acercan y seleccionan a las startups tecnológicas para invertir, concluyendo que aspectos como la capacidad de liderazgo del emprendedor y la calidad / cualidades del equipo humano son importantes, no sólo la tecnología o el retorno financiero previsto. Del mismo modo, Cabiedes (2015) señala que además del modelo 
de negocio y las cifras, para los inversores privados uno de los elementos relevantes para invertir es el equipo que lidera el proyecto. Podría afirmarse que los programas de emprendimiento deben asegurar que los emprendedores conozcan tanto las competencias del perfil emprendedor como las conductas asociadas para lograr mayor eficacia en los proyectos y mayor conciencia de autoeficacia de los emprendedores.

Los programas de emprendimiento buscan principalmente que los beneficiarios comprendan qué es el emprendimiento y pongan en práctica la teoría (Bakotic y Kruzic, 2010), asumiendo que desarrollan los aspectos de la competencia para emprender como son la autoconfianza, la proactividad, la creatividad y el trabajo en equipo (Sánchez-García y Hernández-Sánchez, 2016) aunque su incidencia real en la actividad emprendedora está aún bajo debate.

Safranski (2004) muestra cómo los estudiantes de este tipo de programas presentan diferencias significativas en términos de la tasa de emprendimiento de nuevos negocios, el tamaño de estos y sus ganancias. Sin embargo, algunas investigaciones han concluido que el efecto de este tipo de programas en las habilidades emprendedoras es insignificante y que su impacto sobre la intención de emprender es incluso negativo (Oosterbee et al., 2010). En esta misma línea, Nabi et al. (2017) obtiene resultados con usuarios de los programas que tienen antecedentes familiares o una alta intención emprendedora previa.

Efectivamente, la evaluación del impacto de estos programas es compleja y no debe reducirse a evaluar la tasa de nuevas empresas creadas (Galloway et al., 2015), pues su impacto real se ve a largo plazo y afecta al desarrollo profesional del trabajador en varios aspectos, bien sean empleados o emprendedores (Wilson, 2008). De hecho, consideramos que los emprendedores deberían participar en la definición de las dimensiones de dicha evaluación.

Es necesario orientar a los beneficiarios de estos programas no solo en la fase previa de autodiagnóstico de su perfil emprendedor, sino también durante el proceso de emprendimiento con el fin de aumentar sus competencias emprendedoras y fomentar sus propias estrategias y soluciones a los problemas, más allá del papel de los asesores. Por eso, es conveniente incorporar a los programas de emprendimiento contenidos sobre la elaboración del proyecto profesional y vital.

Efectivamente, las necesidades de orientación personal y profesional en la madurez pueden ser muy variadas. Hernández y Ruiz (2002) afirman que existen diferentes perfiles en las personas desempleadas con necesidades, intereses y expectativas diversas sobre el empleo. Además, el desempleo y la complejidad y constantes cambios del mundo laboral actual pone de manifiesto la necesidad de la orientación profesional, tal y como afirman varios autores (Blasco et al., 2006; Echeverría, 2016; Padilla-Carmona et al, 2014; y otros). Y más en una sociedad como la española cuya tasa de desempleo, según la Encuesta de Población Activa (EPA) del segundo trimestre de 2020, asciende al 15,33\%. 
En cuanto a lo que nos ocupa, la relación del emprendimiento con la orientación profesional, en esta Sociedad del Conocimiento caracterizada por la globalización, la internacionalización y la tecnología, el cambio es la única certeza (Enriques, 2007), y el empleo estable se resiente debido a las necesidades empresariales de adaptar sus producciones a las demandas, lo que provoca el incremento del trabajo flexible en todos los sentidos: flexibilidad de producto (mayor variedad de modelos producidos), flexibilidad funcional (mayor alcance de las tareas realizadas por los trabajadores), flexibilidad numérica (variaciones en la asignación de trabajadores a tareas particulares) (Edgell, 2012) y flexibilidad temporal (producción según la demanda y la oportunidad).

En cuanto al mundo del trabajo, la edad adulta se está reconfigurando como discontinua y reversible' (Loiodice, 2012, p.5) pues, a diferencia de hace unas décadas cuando era posible y habitual desempeñar la misma ocupación durante toda la vida, actualmente las personas se ven obligadas a cambiar de empleo frecuentemente $(\mathrm{Pa}-$ dilla-Carmona, 2001). La rapidez con las que los cambios acontecen es tan elevada que algunos autores como Howkins (2005) consideran que ya estamos pasando de una Sociedad de la Información y el Conocimiento, caracterizada por el boom de las tecnologías de la información, telecomunicaciones, medios de comunicación, servicios financieros, etc., a una sociedad en la que la prioridad está en las ideas y la expresión personal: la Sociedad de la Industria Creativa, que implica trabajo mental y cuyo fruto es la propiedad intelectual. "Si en la primera la tecnología era la reina, en la segunda lo es la imaginación” (Howkins, 2005, p. 117); las ideas, no la información.

Aquí está la clave del emprendimiento: la explotación económica de las ideas y la transformación de ideas en proyectos.

Esta nueva sociedad y sus demandas profesionales generan nuevas necesidades que debe cubrir la orientación profesional. En la actualidad, además del cambio económico hacia un modelo de capitalismo menos regulado, más volátil, basado en el conocimiento y la flexibilidad, y que se mueve a una escala global, aparece la creatividad como factor importante para el mantenimiento de la carrera. Así, los estándares conocidos sobre el funcionamiento del mercado de trabajo se transforman nuevamente en algo desconocido para todos. Howkins (2005) habla del surgimiento de las carreras creativas.

En este sentido, Lingo y Tepper (2013) afirman que los profesionales de sectores donde predomina el autoempleo (y la consecuente autopromoción, el trabajo basado en proyectos, etc.), deben mostrar unos rasgos de personalidad y disposición tales como el profesionalismo, el marketing, la negociación, la sociabilidad, etc., para conseguir emplearse en el sector al que pertenece su profesión. Todos ellos, rasgos asociados a la competencia emprendedora.

Asimismo, el autoempleo y el trabajo por proyectos suele implicar periodos de desempleo más o menos frecuentes, lo que implica que el trabajador sepa gestionar el desempleo como parte de la carrera profesional. 
No es de extrañar que esta sea la tendencia a la que va encaminado el empleo, pues la literatura reciente habla de la desaparición de las carreras lineales y tradicionales (Alcalde, 2004) y del surgimiento de las carreras proteanas (Hall, 2004), sin fronteras (Arthur, 1996), o carreras de portafolios (Cawsey, et al., 1995), que se distinguen de las tradicionales por la necesidad de gestionar la propia carrera y por encontrar la seguridad en la búsqueda del trabajo constante en lugar de la ocupación permanente tal y como vienen haciendo los emprendedores.

Por tanto, para la intervención con emprendedores el reto de la orientación profesional será conseguir adaptarse a sus nuevas demandas. Para ello, al igual que la economía capitalista postindustrial transita hacia un modelo de sociedad basada en la información, el conocimiento y la creatividad, dejando atrás la producción mercantil (donde un buen currículum formativo y experiencial podía dar garantías de una buena posición laboral), debemos redirigir la orientación profesional y emprendedora hacia un modelo que provea a los usuarios de recursos útiles en la actualidad (fomento de la flexibilidad, creatividad y la innovación) para facilitar la mejora de las carreras profesionales de acuerdo a las demandas para emprender.

Este estudio indaga sobre los recursos necesarios para emprender desde el punto de vista de los usuarios de los servicios de emprendimiento. Poniendo especial atención en las necesidades internas que las personas emprendedoras puedan identificar como limitantes de sus iniciativas, creemos necesario abordarlas al objeto de averiguar si es necesario estimular la reflexión personal y el autoconocimiento sobre aquellas actitudes y aptitudes que debe tener un emprendedor para enfrentarse a la aventura de emprender y, en su caso, reforzar o desarrollar las cualidades necesarias.

\section{MÉTODO}

\section{Objetivos}

El objetivo principal de este estudio es identificar qué inhibidores dificultan el emprendimiento de los estudiantes y titulados universitarios de la UNED y cómo abordarlos desde la orientación para la carrera.

Los objetivos específicos derivados del objetivo principal son:

1. Identificar, en los emprendedores universitarios, los motivos que provocan el abandono de sus proyectos de emprendimiento y la mejora de los aspectos personales necesarios para llevarlos a término.

2. Explorar posibles recursos y mejoras que puedan ofertarse a los emprendedores desde los servicios de orientación, a partir de sus demandas. 
Tabla 1. Componentes de la competencia emprendedora desde la perspectiva personal y la empresarial

\begin{tabular}{|c|c|}
\hline Perspectiva empresarial & Perspectiva personal \\
\hline $\begin{array}{l}\text { Conocimiento de los mercados } \\
\text { Habilidad para la gestión de pro- } \\
\text { yectos } \\
\text { Elaborar planes de empresas } \\
\text { Desarrollar modelos de negocios } \\
\text { Conocer procesos de emprendi- } \\
\text { miento } \\
\text { Conocer aspectos empresariales } \\
\text { (finanzas, marketing, etc.) }\end{array}$ & 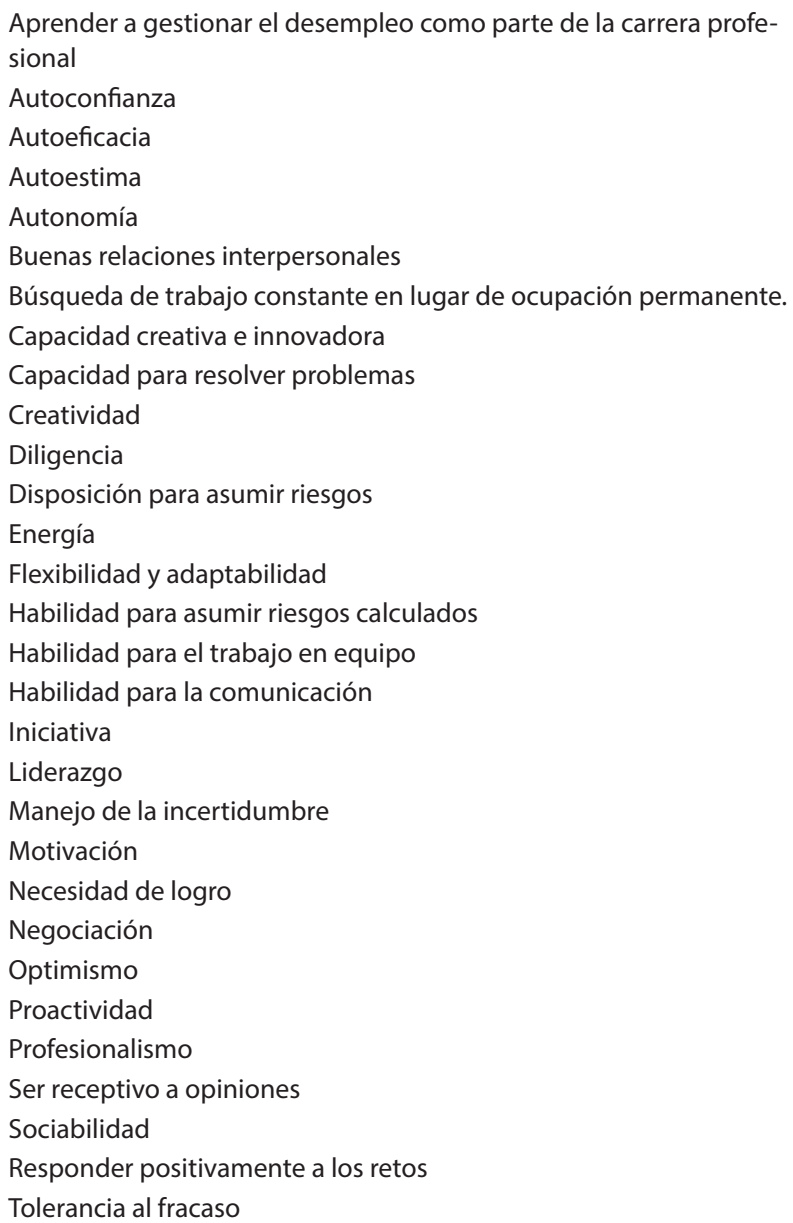 \\
\hline
\end{tabular}

Fuente: elaboración propia

\section{Diseño del estudio}

Se trata de un estudio exploratorio y descriptivo tipo encuesta realizado a estudiantes y titulados de la UNED con intención de emprender o con proyectos en estado incipiente.

Se ha utilizado una metodología cualitativa por ser la más adecuada para identificar las necesidades que experimentan las personas con intención de emprender o con sus proyectos recién iniciados. Como afirma Hernández Sampieri et al. (2010), 
los planteamientos cualitativos sirven como plan de exploración y resultan apropiados cuando buscamos una perspectiva cercana de los participantes.

Para ello, se ha utilizado un cuestionario elaborado ad hoc que permite recabar información sobre los inhibidores para emprender, sobre los aspectos personales que los emprendedores consideran que deben mejorar para emprender, y las ideas y propuestas para mejorar el servicio de orientación para la carrera a emprendedores.

El estudio tiene carácter transversal dado que se recogieron los datos durante la participación de los emprendedores en el 'Programa de emprendimiento universitario 2016-17'.

\section{Participantes}

La población son estudiantes y titulados universitarios con proyectos emprendedores iniciados o con intención de emprender. Se realizó un muestreo no probabilístico intencional (Gil, Rodríguez y García, 1995) que se caracteriza por un esfuerzo deliberado de obtener muestras "representativas" mediante la inclusión en la muestra de individuos supuestamente típicos. Para ello se accedió al registro de las personas solicitantes del curso 'Iniciativa Emprendedora. Proceso de Creación de Empresas' que la Escuela de Organización Industrial (EOI) en colaboración con el COIE de la UNED ofertó gratuitamente a los emprendedores estudiantes y titulados universitarios, durante el curso académico 2016-2017 en los Centros Asociados de la UNED en A-Coruña, Almería, Alzira, Baza, Cádiz, Calatayud, Denia, Vitoria-Gastéiz, Madrid, Pontevedra, Sevilla, Talavera de la Reina, Tenerife, Tortosa, Valdepeñas y Vizcaya. De esta forma, todos los solicitantes del Programa cumplimentaron el cuestionario de tal forma que la muestra obtenida ascendió a 762 sujetos y, por tanto, es coincidente con la población del estudio.

\section{Instrumento}

Se elaboró el cuestionario 'Trayectorias y necesidades de orientación del emprendedor universitario’ con los siguientes bloques de contenido: (Tabla 2)

Los resultados de este tercer bloque se presentan en este artículo.

\section{Análisis de la información}

Para examinar la información recogida en los cuestionarios se ha utilizado la técnica del análisis de contenido (Vallés, 2003), aplicándolo manualmente, en dos 
Tabla 2. Estructura del cuestionario

\begin{tabular}{|c|c|c|}
\hline Bloque & Objetivo & Tipo de preguntas \\
\hline Datos sociodemográficos & $\begin{array}{l}\text { Conocer el perfil del emprendedor } \\
\text { universitario de la UNED }\end{array}$ & \\
\hline Bloque 1 & $\begin{array}{l}\text { Conocer las trayectorias profesionales } \\
\text { y emprendedoras de los participan- } \\
\text { tes }\end{array}$ & $\begin{array}{l}4 \text { preguntas cerradas con tres opcio- } \\
\text { nes de respuesta }\end{array}$ \\
\hline Bloque 2 & $\begin{array}{l}\text { Indagar en el desarrollo de las carac- } \\
\text { terísticas de la persona emprendedo- } \\
\text { ra de los participantes }\end{array}$ & $\begin{array}{l}15 \text { preguntas para valorar con una } \\
\text { escala Likert de cuatro puntos } \\
\text { (4=Mucho/Al máximo; } 3=\text { Lo preciso; } \\
2=\text { =nsuficiente/Poco; } 1=\text { No/Nada) }\end{array}$ \\
\hline Bloque 3 & $\begin{array}{l}\text { Identificar inhibidores para empren- } \\
\text { der que pueden atenderse desde } \\
\text { un enfoque de orientación para la } \\
\text { carrera }\end{array}$ & $\begin{array}{l}2 \text { preguntas para valorar con la } \\
\text { misma escala. } \\
4 \text { preguntas abiertas }\end{array}$ \\
\hline
\end{tabular}

Fuente: elaboración propia

ciclos de análisis y triangulación de investigadores (Valencia, 2000), lo cual permite reducir sesgos y aumentar la comprensión que tenemos del fenómeno de estudio (Benavides y Gómez-Restrepo, 2005), obteniéndose una perspectiva más amplia en el análisis y categorización de datos.

\section{Primer ciclo analítico}

En este primer nivel de análisis, la tarea fue identificar y etiquetar segmentos de texto con significado, para posteriormente codificarlos y jerarquizarlos dentro de un sistema de categorías y subcategorías que conforman la estructura de significados del estudio. Así tras el vaciado de información de cada cuestionario, se procedió a estructurar los datos (Patton, 2002), lo que implicó organizar las unidades, las cate $\theta$ gorías, los temas y los patrones (Grinnell, 1997). Es decir, según en método elemental seguido por Saldaña (2009), cada uno de los investigadores decidió como etiquetaba esa unidad de significado que consideraba relevante. Posteriormente, dentro de este primer ciclo analítico, cada investigador propuso una jerarquización de las etiquetas por temas comenzando la técnica de tematizar datos (Saldaña, 2009).

Una vez etiquetadas las respuestas del primer cuestionario, cada investigador propuso una jerarquización de las etiquetas obtenidas, de forma que cada uno elaboraba una posible estructura del sistema de categorías del contenido de ese cuestio- 
nario en la cual se contemplaban según su criterio: dimensiones, categorías y subcategorías.

La tarea era identificar y etiquetar segmentos de texto con significado a juicio de cada investigador, para posteriormente codificarlos y jerarquizarlos dentro de un sistema de categorías y subcategorías que conformaban la estructura de significados del estudio, como se muestra en la figura 1 .

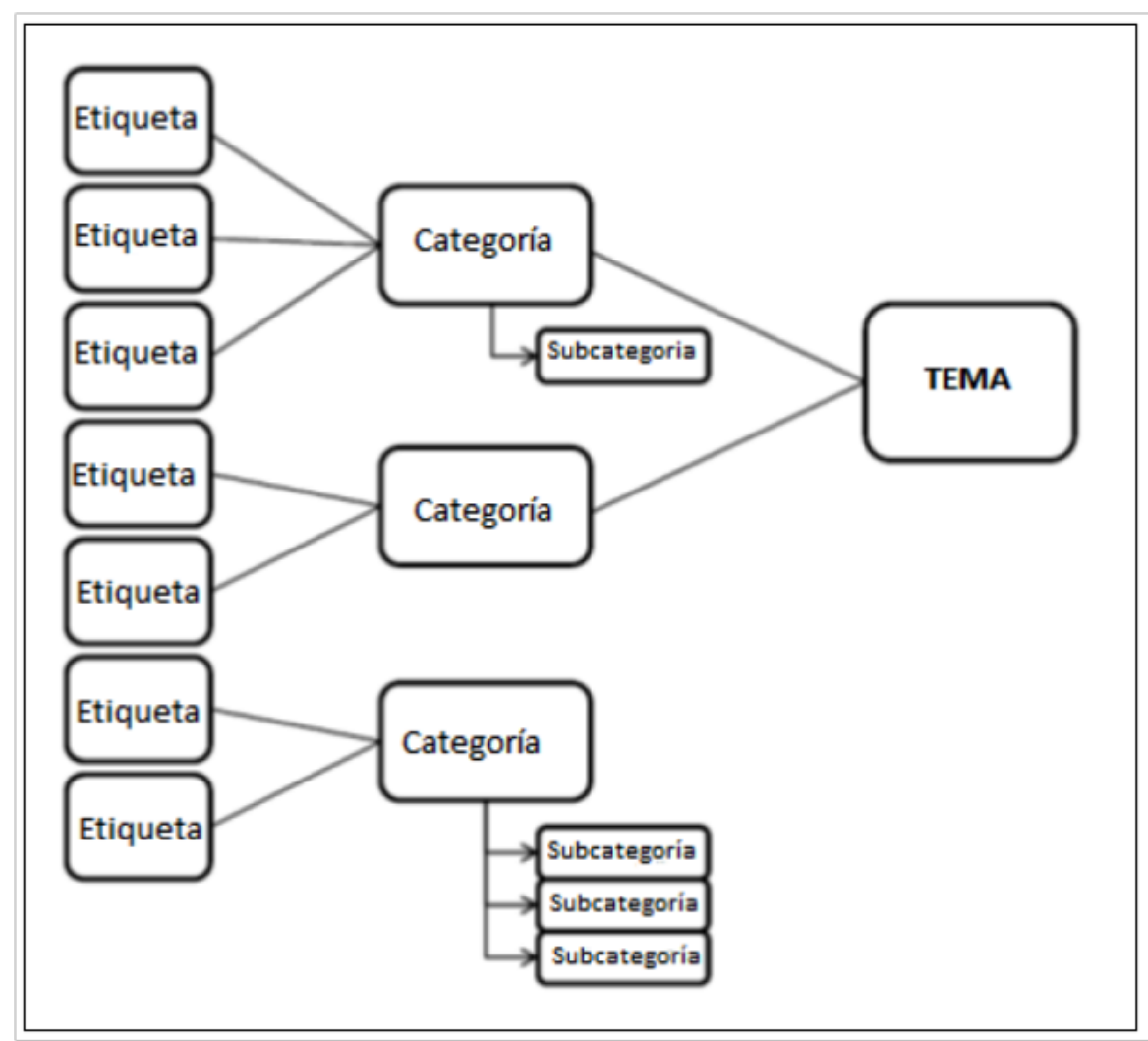

Figura 1. Sistema de categorización en el primer ciclo analítico Fuente: Almagro-Gavira (2017).

Tras el análisis de 20 cuestionarios por cada investigador, se llevó a cabo la primera triangulación de investigadores para poner en común el sistema de categorías, pasando a continuación al segundo ciclo analítico. 
Segundo ciclo analítico

Del análisis de cada cuestionario, surgen distintos sistemas de categorías tentativos que requieren ser unificados para la elaboración del sistema de categorías general. De esta forma, se modifican categorías, surgen nuevas subcategorías, etc., creciendo y consensuándose el sistema tras cada cuestionario analizado. Se comparan las categorías entre sí para agruparlas en temas y buscar posibles vinculaciones entre ellas. Puede afirmarse que este ciclo es más abstracto y conceptual que el primero y de mayor dificultad, pues una vez interpretados los significados de las unidades o fragmentos de textos en el primer ciclo analítico, este segundo implica describir e interpretar el significado de las categorías. Esta actividad conduce al examen de los fragmentos de texto dentro de las categorías, las cuales se disocian de los participantes que las expresaron (Coleman y Unrau, 2005) al cumplimentar el cuestionario.

Posteriormente, se seleccionaron los temas relacionados entre sí que permitieron identificar significados comunes (dimensiones) y las categorías que lo conforman.

Este segundo ciclo analítico supone una forma avanzada de analizar datos y reorganizar los 'códigos' surgidos en el primer ciclo de análisis para desarrollar una síntesis final del sistema de categorías (ver Figura 2). Para ello, se aplicaron los métodos de codificación de modelos, enfocada y axial (Saldaña, 2009).

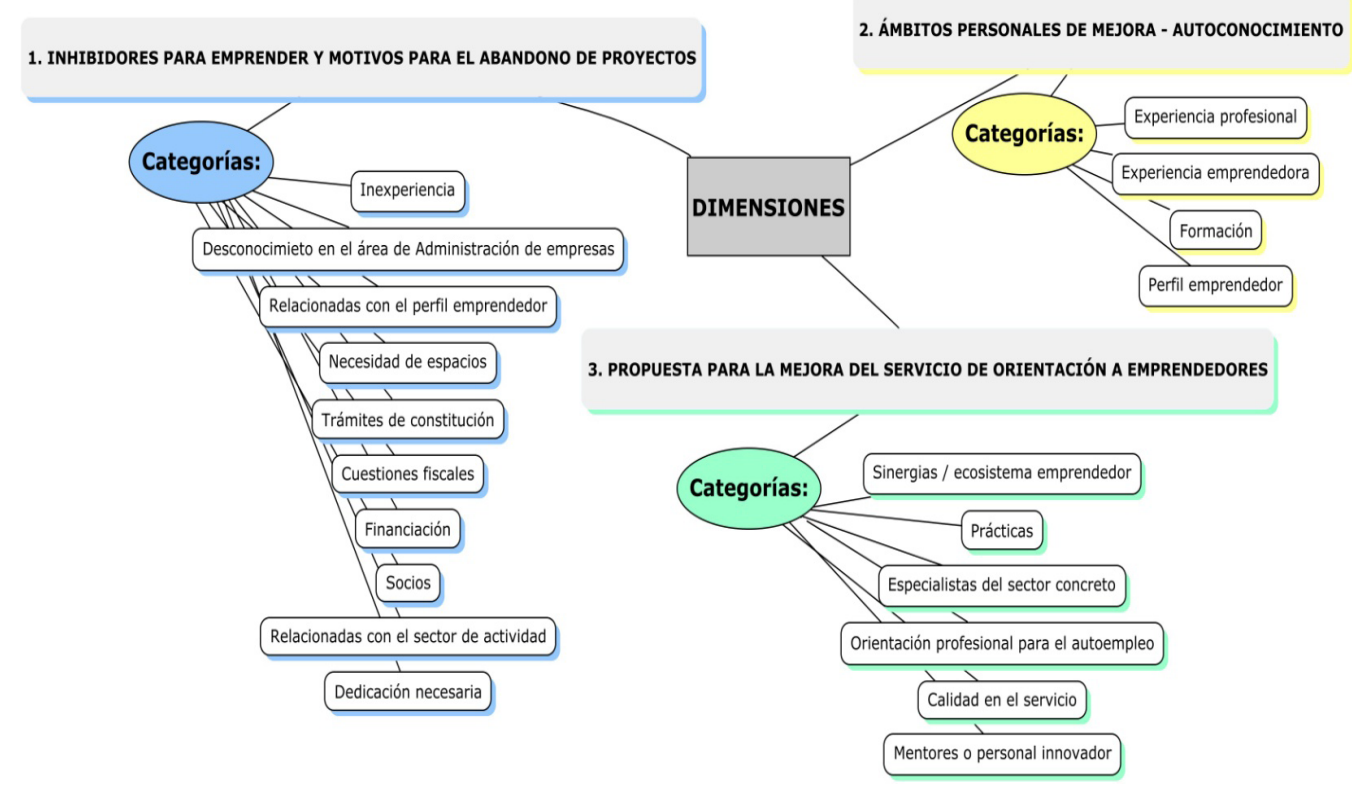

Figura 2. Sistema de categorías general

Fuente: elaboración propia 


\section{RESULTADOS}

Se analizan a continuación los resultados obtenidos en cada una de las dimensiones:

\section{Dimensión 1. Inhibidores para emprender y motivos para el abandono de proyectos}

Se describen las barreras encontradas por las personas con intención de emprender para poner en marcha sus iniciativas o que en algunos casos no consiguen superar una vez iniciado el proyecto y provocan el abandono del mismo. Los impedimentos hallados afectan a los emprendedores desde distintos aspectos, tales como el aspecto material o espacial, el legal o el de la personalidad. En todos los casos, estos factores causan a los emprendedores conflictos con el propósito de emprender que afectan negativamente y frenan el ritmo de sus iniciativas.

Se sintetiza a continuación el contenido de la dimensión 1, cuyas categorías surgen del análisis de las respuestas a las siguientes preguntas: ¿Cuáles han sido los principales inconvenientes que ha encontrado para emprender?, y, En caso de haberse planteado abandonar el proyecto, ¿cuáles han sido los motivos por los que se lo ha planteado?

\section{Categoría 1.1. La inexperiencia}

Los emprendedores consideran que la ausencia de experiencia previa dificulta la toma de decisiones y señalan que liderar proyectos sin la guía y asesoramiento de personas especialistas se muestra como un gran inconveniente para iniciar e impulsar la actividad.

\section{Categoría 1.2. Desconocimiento en el área de Administración de Empresas}

Cuestiones relacionadas tanto con el desarrollo de modelos de negocio sostenibles, los procesos para poner en marcha funciones empresariales, anticipación y análisis de la posible respuesta de los clientes para anticiparse, el precio de los productos o servicios, así como con las tareas administrativas, de contabilidad, etc., frenan la intención de emprender en sus inicios.

\section{Categoría 1.3. Relacionados con el perfil emprendedor}

Hay emprendedores que cuando analizan los inhibidores para emprender se centran en los factores externos a ellos y sus proyectos, de forma que no reconocen la posibilidad de tener obstáculos internos o en sus iniciativas. Desde nuestro punto 
de vista, esto puede ser un importante error que inhibe el emprendimiento. Otros factores relacionados con las deficiencias reconocidas sobre el perfil emprendedor se tratan en la categoría 2.4. "Perfil emprendedor" del bloque 2. "Ámbitos personales de mejora - Autoconocimento".

\section{Categoría 1.4. La falta de tiempo. Dedicación necesaria}

No disponer de tiempo para la dedicación exclusiva ya sea por razones profesionales, estudios, conciliación con la vida familiar, o bien por la concentración de tareas requeridas por la actividad, limita el emprendimiento y supone realmente una barrera para algunos emprendedores.

\section{Categoría 1.5. Necesidad de espacios}

Algunos emprendedores consideran que teniendo un proyecto emprendedor válido, encuentran como principal barrera la falta de espacios gratuitos donde trabajar colaborativamente con el equipo en los primeros momentos del inicio de la actividad en la que no hay ingresos. Esto constituye una barrera importante a la hora de concentrar esfuerzos e impulsar las ideas y el plan de negocio.

\section{Categoría 1.6. Los trámites de constitución y la información y asesoramiento para empezar}

Los emprendedores consideran que la información sobre los requisitos y trámites que deben seguir para dar de alta su actividad, elegir la forma jurídica y constituir una sociedad o darse de alta como autónomo es insuficiente y poco ágil, por lo que requieren asesoramiento.

\section{Categoría 1.7. Fiscalidad}

Las cuestiones relacionadas con la fiscalidad de la actividad, las cuotas a la seguridad social, el coste de los permisos y licencias de aperturas y otros, resultan factores que los emprendedores consideran que inhiben su emprendimiento.

\section{Categoría 1.8. Financiación}

El coste que implica iniciar un emprendimiento y el difícil acceso a la financiación privada es uno de los mayores inconvenientes de las personas emprendedoras. 


\section{Categoría 1.9. Los socios}

Encontrar a otras personas con los mismos intereses e implicación que los promotores, se presenta para los emprendedores como un gran inconveniente para iniciar los proyectos. Las diferencias que surgen en la toma de decisiones, parecen ser otro de los factores que bloquea la correcta marcha de la empresa.

\section{Categoría 1.10. Relacionados con el sector de actividad y con conocimientos técnicos específicos de la actividad a desarrollar}

La ausencia de conocimientos sobre el sector donde quieren operar y en algunos casos, las barreras de entrada que encuentran en dichos sectores les impiden el emprendimiento. En otros, necesitan de conocimientos técnicos específicos sobre la actividad.

\section{Dimensión 2. Ámbitos personales de mejora (auto-identificados)}

Para emprender es necesaria al menos una persona que lidere el emprendimiento. En este sentido la literatura presenta condiciones personales importantes en los individuos que emprenden tales como percibir oportunidades, sopesar los riesgos, el tiempo, los conocimientos, etc., (Varela, 1998; 2001) que son necesarios para emprender. En este bloque proporcionamos información al respecto de los ámbitos personales de mejora que los propios emprendedores identifican.

La dimensión 2 "Ámbitos personales de mejora (auto-identificados)" surge como categoría de la dimensión 1. "Inhibidores para emprender y motivos para el abandono de proyectos". Los temas tratados en esta dimensión tienen relación con las categorías 1.1. "Inexperiencia", 1.2. "Desconocimiento del área de Administración de Empresas", 1.3. "Perfil emprendedor" y 3.1. "Prácticas", la cual corresponde al bloque tercero.

Se sintetiza a continuación el contenido de la dimensión 2 , cuyas categorías surgen del análisis de las respuestas a las siguientes preguntas: ¿En qué ámbitos personales cree que necesita mejorar para llevar a cabo su proyecto de emprendimiento con éxito?; $y_{i}$ Cuáles han sido los principales inconvenientes que ha encontrado para emprender

\section{Categoría 2.1. Experiencia profesional}

La ausencia de experiencia profesional en el sector de actividad donde los emprendedores quieren iniciar sus negocios se identifica como uno de los ámbitos a mejorar reconocido por los emprendedores. 


\section{Categoría 2.2. Experiencia emprendedora}

Algunos emprendedores plantean que la inexperiencia emprendiendo, de ellos mismos y de su entorno cercano, es una diferencia que consideran "negativa" con respecto a otros emprendedores que sí tienen experiencias previas, bien por ellos mismos o por tradición familiar. Así, creen que la experiencia emprendedora previa es un factor considerable a la hora de enfrentarse a futuras dificultades.

\section{Categoría 2.3. Formación}

Además de los aspectos formativos relacionados con la administración de empresas y gestión de negocios presentados en la categoría 1.2. "Desconocimiento en el área de administración de empresas", los emprendedores reconocen necesitar formación en aspectos tales como la inteligencia emocional, y gestión de miedo y la incertidumbre.

\section{Categoría 2.4. Perfil emprendedor}

Algunos emprendedores consideran que su gran obstáculo son ellos mismos, reconociendo que factores como la falta de motivación más allá de la económica, la ausencia de autoconfianza, la inseguridad, tener miedo al fracaso, ser inconstantes, no ser proactivos ni resilientes o no aceptar altos grados de incertidumbre, frenan sus iniciativas. Destaca la existencia de casos de emprendedores potenciales que reconocen no sentirse del todo preparados para emprender, piensan que otros emprendedores son mejores que ellos y, consideran que con un trabajo por cuenta ajena aceptable abandonarían sus proyectos.

\section{Dimensión 3. Propuesta para la mejora del servicio de orientación para la carrera a emprendedores}

Se muestra el conjunto de propuestas que los usuarios emprendedores del COIE han hecho al área de Emprendimiento Universitario. Dichas propuestas abarcan desde una mayor especialización del servicio en orientación y asesoramiento a emprendedores, hasta las distintas acciones que desde su punto de vista contribuirían a la constitución y el mantenimiento sostenido de sus proyectos.

Se sintetiza a continuación el contenido de la dimensión 3, cuyas categorías surgen del análisis de las respuestas a las siguientes preguntas: ¿Qué considera podría hacerse desde los servicios de orientación al emprendimiento para ayudarle a superar las dificultades y/o puntos de inflexión que ha encontrado para emprender? 


\section{Categoría 3.1. Sinergias / ecosistema emprendedor}

Los emprendedores de la UNED consideran útil la creación de una red de cone tactos para colaborar y aprender de otros proyectos, así como para recibir a otros emprendedores interesados en participar en los emprendimientos propios. A tal fin, proponen la creación de una red o ecosistema de emprendedores donde se facilite la difusión de proyectos "en creación", sus necesidades de colaboración, así como la disponibilidad de emprendedores "colaboradores". Asimismo, proponen la creación de eventos de networking para darse a conocer como emprendedores y crear equipos de trabajo.

\section{Categoría 3.2. Prácticas}

Los emprendedores consideran importante las prácticas en su proceso de aprendizaje para el emprendimiento. Piensan que la posibilidad de realizar prácticas en empresas o negocios que estén iniciando su andadura podría darles una amplia visión sobre las necesidades o inconvenientes a los que se deberán enfrentar cuando pongan en marcha sus proyectos, y afirman que no encuentran servicios de emprendimiento que faciliten esa posibilidad. Así, lo proponen como medida para la mejora de estos servicios.

\section{Categoría 3.3. Especialistas del sector concreto}

Los emprendedores proponen dos líneas de mejora relacionadas con la especialidad de los técnicos. Por un lado, consideran que sería de utilidad encontrar técnicos especializados en el emprendimiento social, pues consideran que sus necesidades son diferentes a las de proyectos de negocios y que los técnicos disponibles en los servicios de orientación a emprendedores rara vez saben dar respuesta a sus demandas de información y asesoramiento. Por otro lado, piensan que más allá de las cuestiones genéricas a cualquier proyecto, se encuentran con el desconocimiento por parte de los técnicos del sector o la especialización concreta que se requiere para solventar cuestiones importantes en sus proyectos. Así proponen entre otras medidas que los servicios de emprendimiento se especialicen en sectores o que tengan un plantel de contactos de conocedores de los distintos sectores a quien poder contactar en caso de ser necesario.

\section{Categoría 3.4. Orientación profesional para el autoempleo}

Los emprendedores potenciales afirman que en algunos casos necesitan orientación profesional para tomar la decisión de embarcarse en el emprendimiento. Por otro lado, los emprendedores con negocios en marcha coinciden en tal necesidad afirmando que hubiesen necesitado orientación profesional antes de tomar la deci- 
sión de emprender, e incluso que la necesitan una vez tienen los negocios en funcionamiento. También consideran que aprender a compatibilizar sus cuestiones vitales con las profesionales se les presenta como un gran inconveniente a atender. Asimismo, el deseo o la intención de abandonar el emprendimiento se vuelve transición complicada hasta llevarla o no a efecto, en la cual también les parece necesaria la orientación profesional.

\section{Categoría 3.5. Calidad en el servicio}

Los emprendedores asocian la calidad de los programas de emprendimiento al asesoramiento específico en cuestiones concretas de los proyectos (especializadas), lo cual contraponen a cuestiones genéricas a las que todo emprendedor debe hacer frente y, entienden que otros programas ya atienden. También a la información veraz sobre las implicaciones que supone emprender y a la orientación para la mejora personal y del proyecto, haciendo especial hincapié a las aportaciones que puedan hacer los técnicos a sus proyectos concretos. Asimismo, asocian la calidad del servicio a la agilidad con la que se dan las respuestas a sus consultas, y al seguimiento posterior que se hace de los proyectos una vez se han puesto en marcha, a la facilidad de contactos con inversores o convenios con entidades financieras y, en el caso específico de la UNED, a que desde todos los puntos de atención al emprendedor en la Universidad se atienda con el mismo conocimiento y se pongan a disposición de los emprendedores los mismos recursos.

\section{Categoría 3.6. Mentores o personal innovador}

Los emprendedores de la UNED hacen referencia a la posibilidad de mejorar el servicio ofreciendo desde el mismo la figura de un mentor o guía para su proyecto, que aporte principalmente creatividad o ayuda para ser creativos, y critican la atención reactiva del personal técnico de los servicios, que no incentiva la generación de otras visiones sobre los proyectos.

\section{GONGLUSIONES}

No existen diferencias en cuanto a los motivos que provocan el abandono de los proyectos emprendedores una vez iniciados y los motivos que inhiben o dificultan el emprendimiento a los emprendedores potenciales, pues a nivel general y con la excepción de las barreras de entrada existentes en algunos sectores de actividad, los emprendedores con proyectos en curso identifican los mismos obstáculos que los emprendedores potenciales. Es decir, tal y como afirmaban Manzano y Almagro (2016), utilizando parte de los datos de este estudio, la principal barrera para los emprendedores es la financiación de sus proyectos. "Emprender [...] conlleva unos 
costes de entrada que hay que cubrir, independientemente de si se trata de una decisión motivada por la necesidad o no" (GEM, 2013, p.88). Asimismo, la necesidad de espacios para llevar a cabo sus iniciativas, la burocracia, la alta fiscalidad a la que se ven sometidos y los problemas que surgen por desacuerdos e implicación entre socios ocupan el segundo orden de dificultades.

La experiencia profesional previa en el sector de actividad donde quieren emprender, la experiencia emprendedora entendida como la que proporciona conocimientos prácticos para gestionar negocios, la formación relacionada tanto con la administración de empresas, como, sobre aspectos más personales tales como la inteligencia emocional, la gestión del miedo y la incertidumbre son los ámbitos personales que los emprendedores universitarios consideran que necesitan mejorar. Asimismo, algunos emprendedores consideran que su gran obstáculo es ellos mismos, reconociendo que factores como la falta de motivación más allá de la económica, la ausencia de autoconfianza, la inseguridad, tener miedo al fracaso, ser inconstantes, no ser proactivos ni resilientes o no aceptar altos grados de incertidumbre, frenan sus iniciativas.

Consideramos que desde los programas de emprendimiento debe fortalecerse la capacidad para trabajar sin guía y en soledad. Aprender a "trabajar sin que nadie diga lo que tienes que hacer" (sujeto 241) no parece cuestión sencilla y requiere toda una serie de habilidades personales. Gestionar el qué hacer y cómo hacerlo, se muestra como una habilidad que hay que desarrollar con los emprendedores antes de que éstos se embarquen en sus proyectos.

Destaca la existencia de casos de emprendedores potenciales que reconocen no sentirse suficientemente preparados para emprender, sienten inseguridad respecto a otros emprendedores y reconocen que abandonarían sus proyectos si consiguieran un trabajo por cuenta ajena aceptable. Esto confirma lo anunciado por Aido et al. (2016) cuando afirma que algunos emprendedores se ven forzados a crear negocios para superar barreras externas negativas que no les permiten el desarrollo de otra actividad laboral, siendo además una cuestión que condiciona la continuidad y el éxito de proyectos emprendedores.

Por ello, consideramos que existen diferencias en el éxito emprendedor obtenido según el punto de partida y según la capacidad y preparación auto-percibidas por el emprendedor.

Es un hecho, ya sea por oportunidad o por necesidad, que cada vez más personas deciden emprender como una opción en su carrera profesional, y aunque se sienten inseguras en el proceso, están a la búsqueda de esa idea con la que finalmente lanzarse. Estamos hablando de pre-emprendedores: personas que tienen inquietud por emprender y tienen predisposición a hacerlo (Manzano y Almagro, 2016). 
Normalmente estos pre-emprendedores, que se sitúan en el umbral antes de dar el primer paso para crear su propio proyecto, se centran con o sin ayuda de los programas de emprendimiento en conocer las cuestiones burocráticas y técnicas necesarias en esas primeras fases. Sin embargo, aunque la literatura (Aido et al., 2016) afirma que el emprendedor es la fuente de información fundamental a tener en cuenta para el éxito de su proyecto, desde una perspectiva orientadora es clave que el pre-emprendedor reflexione si está o no preparado para emprender. El deseo y/o la necesidad que tienen los emprendedores vulnerables por emplearse actúa inhibiendo la lógica y lanzándolos a intentarlo, pues comprobamos que pocas veces se realiza un ejercicio de introspección para prever qué se va a necesitar como persona y profesional para llevar a cabo el proyecto.

Por otro lado, los emprendedores de la UNED afirman que los servicios de orientación a emprendedores mejorarían siendo generadores de sinergias y/o ecosistemas emprendedores, si facilitaran la realización de prácticas de emprendimiento, facilitando especialistas del sector de actividad donde los emprendedores quieren emprender, con el fin de solucionar dudas específicas, siendo ágiles en responder a sus demandas específicas, proporcionando orientación profesional además de asesoramiento a los negocios, facilitando información veraz sobre las implicaciones que supone emprender, proporcionando un seguimiento posterior a la puesta en marcha de las iniciativas y, ayudándoles a innovar y a ser creativos con personal capacitado para hacerlo.

Desde su perspectiva, estas cuestiones conformarían un servicio de orientación / asesoramiento a emprendedores de calidad. En este sentido hacemos mención expresa a dos aspectos clave para los programas de orientación del emprendimiento.

Por un lado, se reconoce la importancia de trabajar previamente el autoconocimiento de los emprendedores para saber si están capacitados para emprender o necesitan mejorar sus competencias para afrontar sus proyectos. Así proponemos incluir en los programas de emprendimiento acciones para facilitar el autoconocimiento y explorar las propias fortalezas y debilidades del emprendedor en torno a una idea emprendedora, analizar aspectos actitudinales que pueden influir en el desarrollo de un proyecto emprendedor y adquirir hábitos personales que redunden en una mejora de las capacidades emprendedoras.

Por otro lado, proponemos incluir mejoras en la formación de los técnicos de orientación de emprendedores que les permita ayudarles a innovar y a generar una visión creativa de las iniciativas. 


\section{REFERENCIAS BIBLIOGRÁFICAS}

Aido, B., Diánez, J.P., Carmelo, C. y Ruiz Navarro, J. (2012). Identificación del emprendimiento de alto potencial. Un análisis sobre el reconocimiento de oportunidades en diferentes economías. Enfoques, 339, 75-84.

Alda, R. F. (2010). Perfil Emprendedor de los Estudiantes de la Facultad de Economía y Administración, de la Universidad Católica del Norte, Antofagasta, Chile.Tesis Doctoral, Universidad de Deusto, Facultad de CC.EE. y Empresariales, Bilbao

Alda-Varas, R., Villardón-Gallego, L. y Elexpuru-Albizuri, I. (2012). Propuesta y validación de un perfil de competencias de la persona emprendedora. Implicaciones para la formación. Electronic Journal of Research in Educational Psycology, 10 (3), 1057-1080. DOI: http://dx.doi.org/10.25115/ejrep.v10i28.1549

Almagro-gavira, L.M. y Manzano-Soto, N. (2017, septiembre). Fomento y desarrollo del emprendimiento universitario a través del programa CMETT. Comunicación presentada en AIDIPE, XVIII Congreso Internacional de Investigación Educativa 'Interdisciplinaridad y Transferencia'. Universidad de Salamanca.

Almagro-Gavira, L.M. (2017). El servicio Andalucía Orienta desde la perspectiva de sus usuarios y personal técnico. Factores facilitadores e inhibidores de buenas prácticas en orientación profesional (Tesis doctoral inédita). Universidad de Sevilla, Sevilla

Barba-SÁnchez, V. y Atienza-Sauquillo, C. (2017). Entrepreneurial motivation and self-employment: evidence from expectancy theory. International Entrepreneurship and Management Journal. 13 (4), 1097-1115. DOI: https://doi.org/10.1007/ s11365-017-0441-Z

Bayon, M.C., Lafuente, E. y Vaillant, Y. (2016). Does entrepreneurial confidence influence the nature of entrepreneurial projects chosen by individuals with high ability?. International Journal of Entrepreneurship and Small Business, 26,2, 248-264. DOI: 10.1504/IJESB.2015.071826

BÉCHARD, J.-P. y GRÉGOIRE, D. (2005). Entrepreneurship Education Research Revisited: The Case of Higher Education. Academy of Management Learning y Education, 4(1), $22-43$

Benavides, M.O. y Gómez-Restrepo, C. (2005). Métodos en investigación cualitativa: triangulación. Revista colombiana de Psiquiatría, 34(1), 118-124

Blasco Calvo, P. y Pérez Boullosa, A. (2001). Orientación e inserción profesional: competencias y entrenamiento para su práctica. Valencia: NAU Llibres.

BRIASCO, I. (2014). El desafío de emprender en el S. XXI: Herramientas para desarrollar la competencia emprendedora. Madrid: Narcea Ediciones.

Calvo Gallego, F.J. y Rodríguez-Piñero Royo, M.C. (2011). Colocación y servicios de empleo. Madrid: Consejo Económico y Social.

CÁmara de Comercio (2016). Perfil del emprendedor. ¿Cómo es el emprendedor español? Recuperado de https://www.camara.es/blog/creacion-de-empresas/perfil-del-emprendedor-espanol 
CARsRud, A., y BRÄNnBACK, M. (2011). Entrepreneurial motivations: What do we still need to know? Journal of Small Business Management, 49(1), 9-26. DOI:10.1111/ j.1540-627X.2010.00312.x

Coleman, H., y Unrau, Y.A. (2005). En R. M. Grinnell y Y.A. Unrau (Eds.), Social work: Research and evaluation. Quantitative and qualitative approaches ( $7^{\mathrm{a}} \mathrm{ed}$ ) (pp. 403420). New York: Oxford University Press.

Comisión del parlamento Europeo (2012). Comunicación 795 final de 9 de enero 2012, de la Comisión al Parlamento Europeo, al Consejo, al Comité económico y social europeo y al Comité de las regiones. Plan de acción sobre emprendimiento 2020. Relanzar el espíritu emprendedor en Europa. Bruselas. Recuperado de https:// eur-lex.europa.eu/LexUriServ/LexUriServ.do?uri=COM:2012:0795:FIN:ES:PDF

Corominas, E. (2006). Nuevas perspectivas de la orientación profesional para responder a los cambios y necesidades de la sociedad de hoy. Estudios sobre Educación, 11, 91-110.

Díaz-García, C., SÁEz-Martínez, F. y JiméneZ-Moreno, J. (2015). Evaluación del impacto del programa educativo "Emprendedores" en la intención emprendedora de los participantes. RUSC. Universities and Knowledge Society Journal, 12(3),17-31

Dores Guerreiro, M. y Abrantes, P. (2005). Transiciones a la vida adulta en la era de la globalización. Recorridos de incertidumbre. Recerca, (5), 63-87.

Echeverría, B. (2016). Empleabilidad, Formación y Orientación. En Actas III Congreso Internacional sobre Diagnóstico y Orientación. La orientación Educativa en la Sociedad. Celebrado en Jaén, 8, 9 y 10 de septiembre. Barcelona: Fundación Universitaria Iberoamericana.

EdgelL, S.(2012). The sociology of work: continuity and change in paid andunpaid work ( $2^{\mathrm{a}}$ ed.). Londres: SAGE.

Edwards-Schachter, M., García-Granero, A., Quesada-Pineda, H. y Amara, N. (2015). Disentangling competences: Interrelationships on creativity, innovation and entrepreneurship. Thinking Skills and Creativity,16, 27-39. DOI: https://doi. org/10.1016/j.tsc.2014.11.006

ENRIQues, M.J. (2007). Desarrollo y gestión de carreras con adultos en el siglo XXI: lecturas hacia una armonización de lo global y de lo individual. Revista Electrónica de Investigación Psicoeducativa, 11, (5-1), 75-102.

Eurofound. (2012). Social Partners' Involvement in UnemploymentBenefit Regimes in Europe. Dublín y Bruselas: Fundación Europea para la Mejora de las Condiciones de Vida y de Trabajo.

Farny, S., Frederiksen, S.H., Hannibal, M. y Jones, S. (2016). A Culture of entrepreneurship education. Entrepreneurship \& Regional Development, 28(7-8), 514-535. DOI: https://doi.org/10.1080/08985626.2016.1221228

Galloway, L., Kapasi, I. y Whittam, G. (2015). Exploring 'successful' outcomes of entrepreneurship education: A follow-up study.Industry and Higher Education, 29 (6), 505-515. https://doi.org/10.5367/ihe.2015.0281 
Gálvez, E.J. y García D. (2011). Impacto de la Cultura Intraemprendedora en el rendimiento de las MIPYME del sector turístico colombiano; Un estudio empírico. Universidad y Ciencia, vol. 52, 27-36.

García, M., IbáÑ̃z, J. y Alvira, F. (2000). El análisis de la realidad social: Métodos y técnicas de investigación. Madrid: Alianza.

García-Rodríguez, F. J., Ruiz-Rosa, C.-I., Gil-Soto, E. y Gutiérrez-Taño, D. (2016). Fomento de la educación emprendedora entre el alumnado universitario: diseño y evaluación de un programa de intervención. Cultura y Educación, 28(3), 565-600. DOI: $10.1080 / 11356405.2016 .1196897$

GEM (2012). Global Entrepreneruship Monitor. Informe GEM España 2012. Madrid: Centro Internacional Santander Emprendimiento

GEM (2014). Global Entrepreneruship Monitor. Informe GEM España 2014. Madrid: Centro Internacional Santander Emprendimiento

GEM (2015). Global Entrepreneruship Monitor. Informe GEM España 2015. Madrid: Centro Internacional Santander Emprendimiento.

Gil, J., Rodríguez, G. y García, E. (1995). Estadística Básica Aplicada a las Ciencias de la Educación. Sevilla: Kronos

Hall, P. (2004). Megaciudades, ciudades mundiales y ciudades globales. En A. Martín Ramos (coord.). Lo urbano en 20 autores contemporáneos (pp.117-132). Barcelona: Universitat Politécnica de Catalunya.

Hermansen-Kobulnicky, C.J. y Moss, C.L. (2004). Pharmacy Student Entrepreneurial Orientation: A Measure to Identify Potential Pharmacist Entrepreneurs. American Journal of Pharmaceutical Education, 68 (5), 1-10. DOI: 10.5688/aj6805113

Hernández Fernández, J. y Ruiz del Cerro, J. (2002). La orientación laboral a desempleados inscritos en las oficinas de empleo del INEM. Revista Española de Orientación y Psicopedagogía (REOP). 13, (1), 121-133.

Hernández Sampieri, R., Fernández-Collado, C. y Baptista Lucio, P. (2010). Metodología de la Investigación. México. D.F.: McGraw-Hill.

Kirby, D.A. y Ibrahim, N. (2011). Entrepreneurship education and the creation of an enterprise culture: Provisional results from an experiment in Egypt. International Entrepreneurship and Management Journal, 7(2), 181-193

Ley 6/2007, de 24 de octubre, de Reformas Urgentes del Trabajo Autónomo. Boletín Oficial del Estado núm. 257, de 25 de octubre de 2017.

Lingo, E.L. y Tepper, S.J.(2013). Looking Back, Looking Forward: Arts-Based Careers and Creative Work. Work and Occupations, 40, (4), 337-363.

LiÑán, F. y CHEN, Y.W. (2009). Development and cross-cultural application of a specific instrument to measure entrepreneurial intentions. Entrepreneurship Theory and Practice,33(3), 593-617.DOI: https://doi.org/10.1111\%2Fj.1540-6520.2009.00318.x 
Loiodice, I. (2012). Orientar a las personas adultas en una sociedad compleja. Revista Española de Orientación y Psicopedagogía (REOP), 23 (1), 3-12. DOI: https://doi. org/10.5944/reop.vol.23.num.1.2012.11389

LóPez Sierras, J. (2017). El Canvas del Pre-Emprendedor (beta v1.0). Recuperado de http://www.emotools.com/contents/articulos-y-blogs/juan-lopez-sierras-el-canvas-del-pre-emprendedor/.

Manzano-Soto, N. y Almagro-Gavira, L.M. (2016). Inhibidores para emprender y motivos de abandono de los proyectos de emprendedores universitarios: el caso de la UNED. Comunicación presentada en la Conference of the International Association for Educational and Vocational Guidance (IAEVG) 'Promover la equidad a través de la orientación: reflexión, acción e impacto', junio 2016. Madrid.

Marina, J.A. (2010). La competencia de emprender. Revista de Educación, 351, 49-71.

Martin, B.C., McNally, J.J. y Kay, M.J. (2013). Examining the formation of human capital in entrepreneurship: A meta-analysis of entrepreneurship education outcomes. Journal of Business Venturing, 28(2), 211-224. DOI: https://doi.org/10.1016/j. jbusvent.2012.03.002

Mateos, J.L. (2006). Sociedad del conocimiento. Arbor, vol.182, nº 718, pp.145-151.

Mets, T., Kozlinska, I. y Raudsaar, M. (2017). Patterns in entrepreneurial competences as the perceived learning outcomes of entrepreneurship education: The case of estonian HEIs. Industry and Higher Education, 31(1), 23-33. DOI: https://doi.org/10.1177\%2F0950422216684061

Miller, D. (1983). The correlates of entrepreneurship in three types of firms. Management science, 29(7), 770-791

Nabi, G., Liñán, F., Fayolle, A., Krueger, N. y Walmsley, A. (2017). The Impact of Entrepreneurship Education in Higher Education: A Systematic Review and Research Agenda. Academy of Management Learning y Education, 16(2), 277-299. DOI: https://doi.org/10.5465/amle.2015.0026

Neck, H.M. y Greene, P.G. (2011). Entrepreneurship Education: Known Worlds and New Frontiers. Journal of Small Business Management, 49(1), 55-70. DOI: https:// doi.org/10.1111/j.1540-627X.2010.00314.X

Nielsen, S.L., Nielsen, S.L., Gartner, W.B. y Gartner, W.B. (2017). Am I a student and/or entrepreneur? Multiple identities in student entrepreneurship. Education Training, 59(2), 135-154. DOI: https://doi.org/10.1108/ET-09-2014-0122

OBSERVATORIO DE EMPLEABILIDAD y EMPLEO. COIE-UNED. (2017). Inserción laboral de los titulados de grado 2015. Madrid: UNED.

Oliver, A., Galiana, L. y Gutiérrez-Benet, M. (2016). Diagnóstico y políticas de promoción del emprendimiento en estudiantes. Anales de Psicología, 189-183 ,(1)32. DOI: http://dx.doi.org/10.6018/analesps.32.1.186681 
Oosterbeek, H., van Praag, M. y Ijsselstein, A. (2010). The impact of entrepreneurship education on entrepreneurship skills and motivation. European Economic Review, 54(3), 442-454. DOI: https://doi.org/10.1016/j.euroecorev.2009.08.002

Padilla-Carmona, M.T. (1999). El desarrollo profesional en la edad adulta: Validación del "Adult Career Concerns Inventory" (ACCI) en la población española. (Tesis doctoral). Universidad de Sevilla, Sevilla.

Padilla-Carmona, M.T. (2001). Estrategias para el diagnóstico y la orientación profesional de personas adultas. Barcelona: Laertes

Padilla-Carmona, M.T. (2002). Técnicas e instrumentos para el diagnóstico y la evaluación educativa. ( $2^{a}$ ed.) Madrid: CCS.

Padilla-Carmona, M.T., Suárez, M. y Sánchez, M.F. (2014). Necesidades y competencias de las personas adultas para su inserción profesional. Procedia - Ciencias Sociales y del Comportamiento, 139, 297-304.

PAgÉs, R. (2015). Trabajo creativo como nuevo modelo de trabajo. En San Cornelio, G. (Coord.), Ocupación y Tic en la Sociedad del conocimiento. UOC. Material didáctico no publicado.

Patton, M.Q. (2002). Two decades of developments in qualitative inquiry a personal, experiential perspective. Qualitative social work, 1(3), 261-283. DOI: https://doi. org/10.1177\%2F1473325002001003636

Peñate, W. (1993). Evaluación psicológica: concepto y técnicas de análisis. Valencia: Promolibro.

PÉrez Escoda, N. (1996). Programa de formació per la inserció laboral. Barcelona: CEAC.

Real Decreto 1032/2017, de 15 de diciembre, por el que se aprueba la Estrategia Española de Activación para el Empleo 2017-2020.

Rodríguez, C. (2003). Nociones y destrezas básicas sobre el análisis de datos cualitativos. El proceso de Investigación en educación, algunos elementos claves. Granada: MIDE Universidad de Granada.

SAFrAnski, S.R. (2004). Resource Reviews. Academy of Management Learning and Education, 3(3), 340-342. https://doi.org/10.5465/amle.2002.7373750

Saldaña, J. (2009). The Coding Manual for Qualitative Researchers.London: Sage.

Sánchez García, M.F. (2004). Orientación laboral para la diversidad y el cambio. Madrid: Sanz y Torres.

Sánchez García, M.F. (2012). Principios, contextos y dimensiones principales de intervención en orientación profesional. En M.F. SÁNCHEZ García y M. Álvarez GonzÁlez (Coord.). Bases teórico-prácticas de la orientación profesional. Madrid: UNED (Cap. II, pp. 54-79).

Sánchez-García, J.C y Hernández-Sánchez, B.R. (2016). Influencia del Programa Emprendedor Universitario (PREU) para la mejora de la actitud emprendedora. 
Pampa. Revista Interuniversitaria de Estudios Territoriales, 12(13), 55-75. DOI: http://dx.doi.org/10.14409/pampa.v0i13.5906

SEwell, P. y Pool, L. D. (2010). Moving from conceptual ambiguity to operational clarity: Employability, enterprise and entrepreneurship in higher education. Education + Training, 52(1), 89-94. DOI: https://doi.org/10.1108/00400911011017708

Sobrado, L. y Fernández, E. (2010). Competencias emprendedoras y desarrollo del espíritu empresarial en los centros educativos. Educación XX1, 13(1), 15-38. DOI: https://doi.org/10.5944/educxx1.13.1.275

ŠTefanić, I., Šimunović, G., ŠTefanić, E., y CAMPbell, R.K. (2017). Entrepreneurial education for students of non-economics educational programs. Tehničkivjesnik, 24(4), 1079-1086. DOI: https://doi.org/10.17559/TV-20161128153529

Stuckler, D. y Basu, S. (2013). Por qué la austeridad mata. El coste humano de las políticas de recorte. Madrid: Taurus.

Suárez-Ortega, M. Sánchez-García M.F. y García-García, M.C. (2016). Caracterización de buenas prácticas y necesidades de mejora en los servicios de orientación para el empleo. Revista Española de Orientación y Psicopedagogía (REOP), vol. 27, 3, 43-60. DOI: https://doi.org/10.5944/reop.vol.27.num.3.2016.18799

Torrejón, C. (2016). Perfiles competenciales y éxito emprendedor. Implicaciones para los inversores en Capital Riesgo. Revista Española de Capital Riesgo. 2, 29-47.

Uy, M.A., Foo, M.D., Y ILIES, R. (2015). Perceived progress variability and entrepreneurial effort intensity: The moderating role of venture commitment. Journal of Business Venturing, 30(3), 375-389. DOI: https://doi.org/10.1016/j.jbusvent.2014.02.001

VALENCIA, M.A. (2000). La triangulación metodológica: sus principios, alcances y limitaciones. Investigación y educación en enfermería, 18(1), 13-26.

VAllejo, R. y Finol de Franco, M. (2010). La triangulación como procedimiento de análisis para investigaciones educativas. Redhecs, 7(4), 117-133. Recuperado de http://publicaciones.urbe.edu/index.php/REDHECS/article/viewArticle/620

VAllés, M. S. (2003). Técnicas cualitativas de investigación social. Madrid: Síntesis.

VAn PraAg, C.M., y Versloot, P.H. (2007). What is the value of entrepreneurship? A review of recent research. Small Business Economics, 29(4), 351-382. DOI: https:// doi.org/10.1007/s11187-007-9074-X

VARELA, R. (1998). Innovación empresarial. Un nuevo enfoque de desarrollo (5a ed.), ICESI: Cali, Colombia.

VÉlaz de Medrano, C. (2008). Formación y profesionalización de los orientadores desde el enfoque de competencias. Educación XXI, 11, 155-181. DOI: https://doi. org/10.5944/educxx1.11.0.313

Welch, C., Piekkari, R., Plakoyiannaki, E. y Paavilainen-Mantymaki, E. (2011). Theorising from case studies: Towards a pluralist future for international business 
Inhibidores para emprender... - L.M. Almagro-Gavira y N. Manzano-Soto

research. Journal of International Business Studies, 42(5), 740-762. DOI: https://doi. org $/ 10.1057 /$ jibs.2010.55

Wilson, K. E. (2008). Entrepreneurship education in Europe. Entrepreneurship and higher education. Chapter 5, OECD. Recuperado de https://ssrn.com/abstract $=1392369$

Yedigis, B.L. y Weinback, R.W (2005). Using existing knowledge. En R.M. Grinnell y Y.A. Unrau (Eds.) Social work: Research and evaluation. Quantitative and qualitative aproaches ( $7^{\mathrm{a}} \mathrm{ed}$.) (pp. 45-57). Nueva York: Oxford University Press. 